\title{
Long-Term Potentiation Enhances Neurogenesis in the Adult Dentate Gyrus
}

\author{
Elodie Bruel-Jungerman, ${ }^{1}$ Sabrina Davis, ${ }^{1}$ Claire Rampon,,${ }^{1,2}$ and Serge Laroche ${ }^{1}$ \\ ${ }^{1}$ Laboratoire de Neurobiologie de l'Apprentissage, de la Mémoire et de la Communication, Centre National de la Recherche Scientifique Unité Mixte de \\ Recherche 8620, Université Paris-Sud, 91405 Orsay, France, and ${ }^{2}$ Centre de Recherches sur la Cognition Animale, Centre National de la Recherche \\ Scientifique Unité Mixte de Recherche 5169, Université Paul Sabatier, 31062 Toulouse, France
}

Activity-dependent synaptic plasticity and neurogenesis are two forms of brain plasticity that can participate in functional remodeling of neural networks during the formation of memories. We examined whether long-term potentiation (LTP) of excitatory synaptic transmission, a well characterized form of synaptic plasticity believed to play a critical role in memory formation, can regulate the rate of neurogenesis in the adult rat dentate gyrus in vivo. We first show that induction of LTP at medial perforant path-granule cell synapses stimulates the proliferation of progenitor cells in the dentate gyrus with a consequential long-term persistence of a larger population of surviving newborn cells. Using protocols to examine the effect of LTP on survival, we next show that LTP induction promotes survival of 1- to 2-week-old dentate granule cells. In no case did LTP appear to affect neuronal differentiation. Finally, we show that LTP induces expression of the plasticity-related transcription factor Zif268 in a substantial fraction of 2-week-old but not 1-week-old neurons, suggesting the prosurvival effect of LTP can be observed in the absence of LTP-mediated Zif268 induction in newborn cells. Our results indicate that electrically induced LTP in the dentate gyrus in vivo provides a cellular/molecular environment that favors both proliferation and survival of adult-generated neurons.

Key words: synaptic plasticity; memory; hippocampus; cell survival; Zif268; transcription factor

\section{Introduction}

The formation of memories relies on activation of several molecular cascades in neurons that ultimately lead to long-lasting changes in synaptic structure and function. To date, the idea that activity-dependent synaptic plasticity modeled experimentally by long-term potentiation (LTP) plays a central role in the establishment of memories has received considerable support. For instance, in the hippocampus and dentate gyrus (DG), there is evidence that mechanisms underlying LTP of excitatory synapses are activated during learning and required for the laying-down of several forms of hippocampal-dependent memories (Lynch, 2004). However, beyond this mechanism of synaptic plasticity, in defined brain regions such as the DG of the hippocampus, adult neurogenesis, the birth and growth of new neurons, is another form of neural plasticity that may contribute to hippocampaldependent memory function (Shors et al., 2001; BruelJungerman et al., 2005; Winocur et al., 2006). In the DG, new granule cells (DGCs) are generated from progenitor cells originating in the subgranular zone (SGZ), where neurogenic activity

\footnotetext{
Received Feb. 21, 2006; revised April 20, 2006; accepted April 23, 2006.

This work was supported by the Centre National de la Recherche Scientifique, by European Union Grant RTN-CT2003-504231 to S.L., by an Institut Universitaire de France grant, by the Fondation NRJ-Institut de France (C.R.), and by a fellowship from the Fondation pour la Recherche Médicale to E.B.-J. We thank Isabelle Massou for excellent technical assistance with pCREB analysis and IFR46 for access to confocal microscope.

Correspondence should be addressed to Dr. Serge Laroche, Laboratoire de Neurobiologie de l'Apprentissage, de la Mémoire et de la Communication, CNRS UMR 8620, Université Paris-Sud, Bâtiment 446, 91405 Orsay, France. E-mail: serge.laroche@ibaic.u-psud.fr.

D0I:10.1523/JNEUROSCI.0782-06.2006

Copyright $\odot 2006$ Society for Neuroscience $\quad$ 0270-6474/06/265888-06\$15.00/0
}

is maintained throughout life (Kempermann et al., 2003). It is now well established that although many new neurons fail to reach maturity and die within a few weeks of their birth, a significant fraction mature into functional DGCs, extend axons toward CA3 pyramidal cells and dendritic arborizations in the molecular layer, becoming fully functional and synaptically integrated into existing circuits of mature neurons (van Praag et al., 2002; Zhao et al., 2006).

Several reports have demonstrated that behavioral activity such as environmental enrichment (Kempermann et al., 1997; Bruel-Jungerman et al., 2005), physical exercise (van Praag et al., 1999), and specific hippocampal-dependent learning tasks (Gould et al., 1999; Snyder et al., 2005) can regulate neurogenic activity in the DG. Enrichment and voluntary running also facilitate hippocampal LTP and improve learning (van Praag et al., 1999; Duffy et al., 2001), and learning itself induces LTP-like mechanisms (Martin and Morris, 2002; Lynch, 2004). Thus, although synaptic plasticity and neurogenesis may have independent contributions to hippocampal functions, correlational studies suggest a possible coupling between synaptic plasticity and neurogenesis. In other words, is it possible that synaptic plasticity per se might influence adult neurogenesis? We approached this issue by analyzing the effect of LTP on neurogenesis and show that LTP induction can effect both proliferation of progenitor cells and survival of recently born DGCs.

\section{Materials and Methods}

Subjects. Adult male Sprague Dawley rats (220-250 g; Charles River, Orléans, France) were housed in pairs in a temperature-controlled col- 
ony room $\left(25^{\circ} \mathrm{C}\right)$ on a $12 \mathrm{~h}$ light/dark cycle, with ad libitum access to food and water. All experiments were conducted in accordance with the European Union (86/609/EEC) and the French National Committee (87/ 848) guidelines.

Electrophysiology. We induced LTP in the DG unilaterally, each rat serving as its own control, using surgical and LTP-inducing procedures described previously (Davis et al., 2000). Briefly, under pentobarbital anesthesia (60 mg/kg, supplemented as required) a stimulating electrode was placed in the angular bundle of the perforant path and a recording electrode in the hilus of the DG. A 30 min baseline was recorded under low-frequency stimulation $(0.033 \mathrm{~Hz})$ followed by delivery of a tetanus [six series, $2 \mathrm{~min}$ apart, of six trains of pulses $(400 \mathrm{~Hz}, 20 \mathrm{~ms})$ at $10 \mathrm{~s}$ intervals] to induce LTP, or a pseudotetanus (six series, 2 min apart, of six pulses at $10 \mathrm{~s}$ intervals) to mimic this pattern of stimulation without inducing LTP. The stimulus intensity was kept constant throughout. Evoked responses recorded before and after $(1 \mathrm{~h})$ LTP induction or pseudotetanus were stored for analysis of fEPSP slope and population spike amplitude (Davis et al., 2000). Responses were normalized to baseline and data were analyzed using ANOVA. At the end of the experiment, electrodes were removed, the skin was sutured and rats were returned to their home cages.

BrdU injection protocols. To investigate the effect of LTP on the proliferation of progenitor cells and their later survival, rats were given three injections of 5-bromo-2'-deoxyuridine (BrdU; Sigma, St. Louis, MO) [2 h apart, $75 \mathrm{mg} / \mathrm{kg}$ i.p., dissolved in $0.9 \% \mathrm{NaCl}$ and $0.007 \mathrm{~N} \mathrm{NaOH}(10$ $\mathrm{mg} / \mathrm{ml}$ )] either 1 or $4 \mathrm{~d}$ after LTP induction. They were anesthetized and perfused with $4 \%$ paraformaldehyde, $24 \mathrm{~h}$ (proliferation) or 4 weeks (long-term persistence) after BrdU injections. To examine the effect of LTP on cell survival, rats were first injected with BrdU $(75 \mathrm{mg} / \mathrm{kg}$, once a day for $3 \mathrm{~d}$ ) and LTP was induced 1 or 2 weeks later. Rats were then perfused 4 weeks after BrdU injections. In additional groups, rats were perfused $2 \mathrm{~h}$ after LTP induction. Brains were postfixed overnight and transferred into $30 \%$ sucrose. Serial coronal sections cut on a cryostat $(30$ $\mu \mathrm{m})$ were stored in cryoprotectant at $-20^{\circ} \mathrm{C}$.

Immunohistochemistry. Peroxidase immunolabeling of $\mathrm{BrdU}$ was performed as described previously (Bruel-Jungerman et al., 2005). One-insix sections throughout the DG were denaturated $(2 \mathrm{~N} \mathrm{HCl})$, incubated overnight in mouse monoclonal anti-BrdU (1:1500, MAB 3424; AbCys, Paris, France), and then in biotinylated goat anti-mouse antiserum (1: 200). Sections were counterstained with Nuclear fast red (Vector Laboratories, Burlingame, CA) allowing DG volume analysis. For immunohistofluorescence, one in 12 sections were denaturated, followed by overnight incubation in monoclonal anti-BrdU (1:400, OBT0030; AbCys) alone or simultaneously with rabbit polyclonal anti-Prox-1 (1:1800; Chemicon, Temecula, CA) or rabbit polyclonal anti-phosphorylated cAMP response element-binding protein $\left[\mathrm{pCREB}^{\left(\operatorname{ser}^{133}\right)}\right.$ (1:400; Upstate Biotechnology, Lake Placid, NY). Sections were incubated in biotinylated goat anti-rat antiserum (1:400; Vector Laboratories), then streptavidin Alexa-568 (1:800; Invitrogen, Eugene, OR) alone, or with Alexa-488 goat anti-rabbit antiserum (1:200; Invitrogen). BrdU-alonestained sections were incubated overnight in mouse monoclonal antiNeuN (1:500; Chemicon) or rabbit polyclonal anti-Zif268 (1:1250; Santa Cruz Biotechnology, Tebu, France), and in Alexa-488 goat anti-mouse highly cross-adsorbed antiserum or Alexa-488 goat-anti-rabbit (1:200; Invitrogen).

Quantification and image analysis. Stereological quantification of BrdU-labeled nuclei was conducted in LTP and control sides of the dorsal DG (20 sections per rat; bregma -2 to -5.4 ) as described previously (Bruel-Jungerman et al., 2005). To avoid oversampling errors, nuclei intersecting the uppermost focal plane were excluded. Absolute numbers of BrdU-labeled cells were obtained by multiplying BrdU-cell density by the reference volume. For double-labeling, percentages of BrdU-labeled nuclei coexpressing Prox-1, NeuN, pCREB, or Zif268 were determined by analyzing 50-100 randomly selected BrdU-labeled nuclei throughout the granule cell layer and SGZ of dorsal DG using a Zeiss (Oberkochen, Germany) confocal microscope. BrdU-positive nuclei were analyzed ( $63 \times$ oil objective) in their entire $z$-axis $(0.5 \mu \mathrm{m}$ steps $)$ and were rotated in orthogonal planes $(x-y)$ to verify double-labeling and exclude false double-labeling caused by overlay of signals from different cells. Analyses were performed in sequential scanning mode to rule out cross-bleeding between detection channels. ANOVA and $t$ tests (nonpaired or paired when comparing the control and LTP sides) were used for statistical analyses.

\section{Results \\ LTP enhances proliferation and later survival of newly generated DGCs}

To examine the effect of LTP on cell proliferation, LTP was induced by tetanic stimulation of the medial perforant path in vivo, followed by injections of BrdU 1 or 4 d later. Tetanic stimulation resulted in rapid and stable potentiation of the field EPSP (fEPSP) slope (45.6 $\pm 5.5 \% ; p<0.0001)$ (Fig. $1 a)$ and population spike amplitude ( $438.5 \pm 91.6 \% ; p<0.001$; data not shown), whereas there was no change with pseudotetanus (Fig. 1a) (fEPSP, $1.52 \pm$ $3.93 \%$; spike, $63.07 \pm 21.79 \%$; between-group difference, $\left.F_{(1,23)}=36.9 ; p<0.0001\right)$. In all experiments reported here, measurements of DG volumes confirmed no effect of LTP $\left(F_{(1,36)}=0.44 ; p=0.51\right)$. Proliferation of progenitor cells was examined $24 \mathrm{~h}$ after BrdU injections. Quantitative analysis revealed that the number of BrdU-labeled cells in the DG was significantly greater on the LTP side compared with the contralateral side $\left(n=8 ; t_{(7)}=3.16 ; p=0.016\right)$ (Fig. $\left.1 b\right)$. Typically, LTP resulted in a large increase in clustered BrdU-labeled nuclei in the SGZ (Fig. 1c). The promitotic effect of LTP was more pronounced at $4 \mathrm{~d}\left(t_{(14)}=2.29 ; p=0.038\right)$, with a $78 \%$ increase in the number of proliferating cells $(n=8 ; 1252.6 \pm 178.6$ and $2140.1 \pm 307.2$ in control and LTP sides, respectively; $t_{(7)}=4.54$; $p=0.0027$ ) (Fig. 1b). Control experiments showed this effect was not caused by implantation of electrodes, synaptic activation, or DGCs discharges because no change in BrdU-labeled cell numbers was observed after pseudotetanus (Fig. $1 b)(n=7 ; t$ test, $p>$ $0.5)$. ANOVA confirmed a significant increase in BrdU-labeled cell numbers after LTP, compared with the pseudotetanus $\left(F_{(1,13)}\right.$ $=11.12 ; p=0.005$; group $\mathrm{x}$ treatment interaction, $F_{(1,13)}=16.0$, $p=0.0015 ; t$ test on treatment: $\left.t_{(13)}=2.63 ; p=0.021\right)$. In these two groups, newborn cells were also analyzed for coexpression of BrdU and pCREB and BrdU and Prox-1 (Fig. 1d), an early expressed neural lineage-specific homeobox transcription factor selective to DGCs (Kronenberg et al., 2003). There was no significant difference in BrdU/pCREB coexpression between the LTP $(56.0 \pm 4.5 \%)$ and pseudotetanus $(48.8 \pm 5.7 \%)$ groups $\left(t_{(6)}=\right.$ $0.99 ; p=0.36)$ and these values were not different from the control sides $(62.0 \pm 1.8$ and $56.7 \pm 6.0 \%$, respectively). Despite enhanced cell proliferation with LTP, the percentage of cells coexpressing BrdU/Prox-1 was similar in LTP $(78.1 \pm 4.1 \%)$ and pseudotetanus $(72.6 \pm 3.5 \%)$ groups $\left(t_{(8)}=1.02 ; p=0.34\right)$. In all, these results show that induction of LTP significantly enhances proliferation of DG progenitor cells without affecting differentiation.

We next examined the long-term fate of mitotically active cells by injecting BrdU $4 \mathrm{~d}$ after LTP and killing the rats 4 weeks later $(n=11)$. In line with the reported death of newborn cells with time (Gould et al., 1999), the number of BrdU-positive nuclei in control sides at 4 weeks $(829.2 \pm 118.3)$ had declined significantly compared with that at $24 \mathrm{~h}(1268.5 \pm 100.8)($ Fig. $1 b)\left(F_{(2,23)}=\right.$ $3.85 ; p=0.036$, compared with LTP and pseudotetanus control sides; post hoc comparisons, $p<0.05$ in each case). Quantitative analysis showed the number of BrdU-labeled nuclei on the LTP side $(1704.5 \pm 264.1)$ was strongly increased 4 weeks after BrdU injections compared with the contralateral side $\left(t_{(10)}=4.08 ; p=\right.$ 0.0022 ) (Fig. 1b,e), indicating a net beneficial effect of LTP on survival. However, a nonsignificant group x treatment interac- 

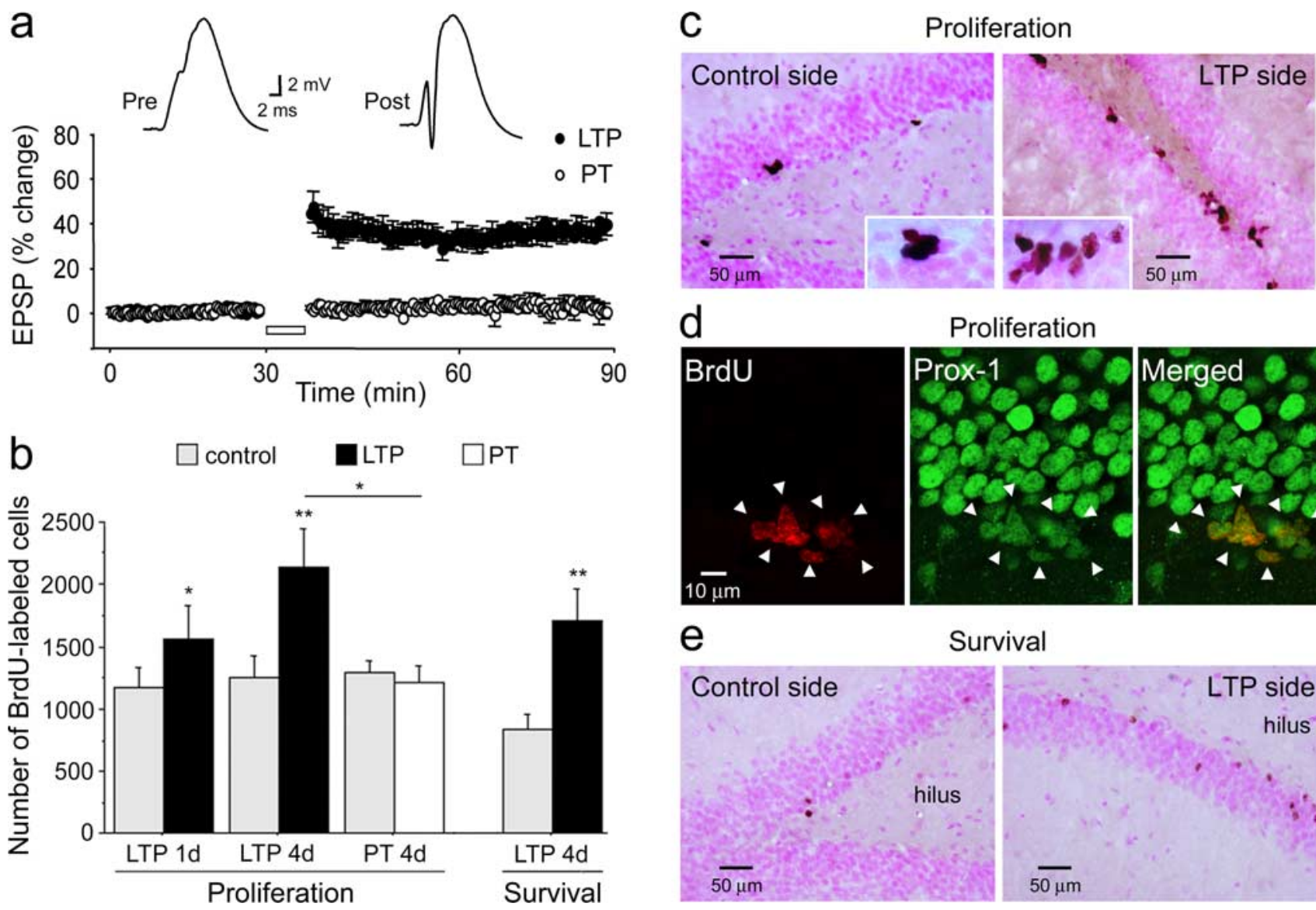
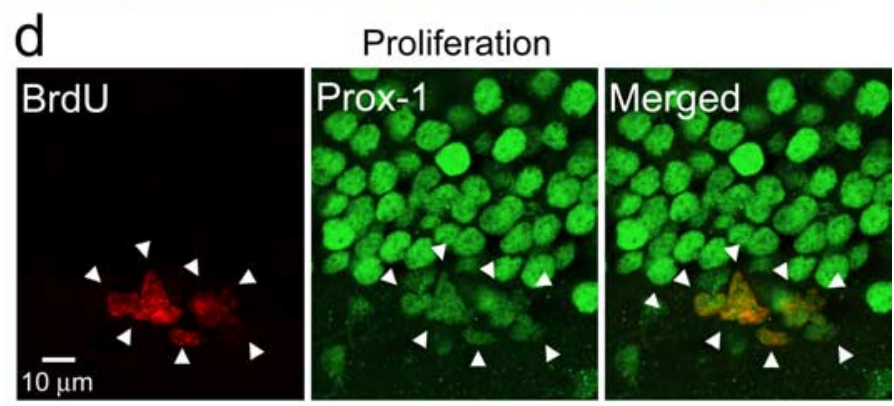

e

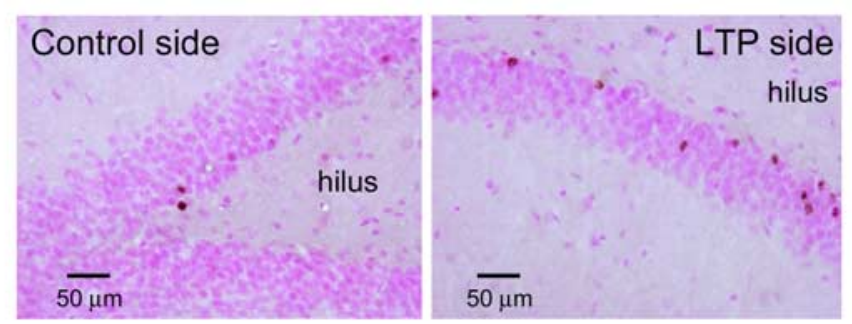

Figure 1. LTP enhances proliferation and later survival of newborn cells in vivo. $\boldsymbol{a}$, Perforant path tetanus (bar) induced stable LTP of the fEPSP, whereas pseudotetanus (PT) had no effect. Evoked potentials recorded before and after LTP are shown above. $\boldsymbol{b}$, Average total number of BrdU-labeled cells (mean \pm SEM) in the DG from the control, LTP, and PT sides. LTP was induced $1 \mathrm{~d}$ or $4 \mathrm{~d}$ before BrdU injections and rats were killed $24 \mathrm{~h}$ (proliferation) or 4 weeks (survival) later. ${ }^{*} p<0.05 ;{ }^{* *} p<0.005$. c, Representative images of sections (control and LTP sides) stained for BrdU (dark) with nuclear fast red counterstaining (pink) from an animal injected with BrdU $4 \mathrm{~d}$ after LTP induction and killed $24 \mathrm{~h}$ after. Insets, Illustrations of clusters. $\boldsymbol{d}$, Confocal images of BrdU (red) and early neuronal marker Prox-1 (green) immunohistochemistry illustrating colocalization (merged) in DGCs (arrowheads). $\boldsymbol{e}$, LTP induction $4 \mathrm{~d}$ before BrdU injections resulted in more BrdU-labeled cells at the 4 week survival time.

tion $\left(F_{(1,17)}=0.002 ; p=0.97\right)$ comparing the number of BrdUlabeled nuclei present $24 \mathrm{~h}$ and 4 weeks after BrdU injections suggests the observed prosurvival effect of LTP on cells labeled $4 \mathrm{~d}$ after LTP is likely to be a direct reflection of LTP-induced increase in the overall population of proliferating cells.

LTP enhances the survival of 1- and 2-week-old cells

To specifically address the issue of whether LTP induction can affect survival of newborn cells in the DG, we first injected rats with BrdU (once a day for $3 \mathrm{~d}$ ), induced LTP 1 or 2 weeks later, and then quantified surviving BrdU-labeled cells 4 weeks after the last BrdU injection. Time points of 1 and 2 weeks were chosen to correspond with a window of maximal death rate of newborn DGCs (Gould et al., 1999). Again, induction of LTP was robust and stable (fEPSP, $46.3 \pm 6.5 \%$; $p<0.0001$ ) (Fig. $2 a$ ) and comparable with that of the previous experiment, and there was no significant change in fEPSP $(1.61 \pm 11.9 \%)$ or spike $(12.92 \pm$ $20.55 \%$ ) in the pseudotetanus group (Fig. $2 a$ ). Four weeks after BrdU injections, the number of surviving BrdU-positive cells when LTP was induced 1 week $(1272 \pm 123.5 ; n=6)$ or 2 weeks (1258.7 $\pm 92.0 ; n=8)$ post-BrdU was significantly increased compared with the respective contralateral sides $(871 \pm 66.8$ and $1034.3 \pm 66.9$; LTP effect: $t_{(5)}=6.56, p=0.0012$ and $t_{(7)}=4.13$, $p=0.0044$ at 1 and 2 weeks, respectively) (Fig. $2 b, c)$. No change was observed in control rats receiving a pseudotetanus (Fig. $2 b$ )
$(990.4 \pm 151.0$ and $904.5 \pm 116.2$ respectively; $n=4$; $\left.t_{(3)}=1.45 ; p=0.24\right)$, confirming that enhancement of survival was attributable to synaptic plasticity. ANOVA confirmed a significant prosurvival effect of LTP compared with the pseudotetanus (group $\mathrm{x}$ treatment interaction, $F_{(1,10)}=12.39, p=0.0055$; LTP vs pseudotetanus sides, $\left.t_{(10)}=2.29, p=0.045\right)$. Percentages of BrdU/pCREB coexpressing cells were higher than when animals were killed $4 \mathrm{~d}$ after BrdU (see above, LTP enhances proliferation and later survival of newly generated DGCs); again, however, we found no difference between the LTP $(88.7 \pm 3.9 \%)$ and pseudotetanus $(93.2 \pm 1.9 \%)$ groups $\left(t_{(6)}=1.05 ; p=0.335\right)$, and no difference with the control sides $(89.5 \pm 1.0 \%$ and $92.3 \pm$ $1.0 \%$, respectively).

As expected, confocal analysis of colocalization of BrdU with the neuron-specific marker NeuN (Fig. 2e) showed that the majority of BrdU-positive cells ( $\sim 75 \%)$ expressed a neuronal phenotype. We found no evidence that LTP affected differentiation when induced 1 week $(69.8 \pm 3.7 \%$ and $70.8 \pm 6.7 \%$ in the control and LTP sides, respectively; $\left.t_{(3)}=0.16 ; p=0.89\right)$ or 2 weeks $\left(79.3 \pm 2.8\right.$ and $\left.82.9 \pm 3.8 \% ; t_{(3)}=1.93 ; p=0.15\right)$ after BrdU injection (LTP effect: $F_{(1,6)}=0.49, p=0.51$; interaction: $\left.F_{(1,6)}=0.16, p=0.71\right)$. Thus, LTP does not influence neuronal determination of cells born 1 or 2 weeks before, confirming that neuronal commitment is an early step in the maturation process of newly generated DGCs (Kempermann et al., 2003). 

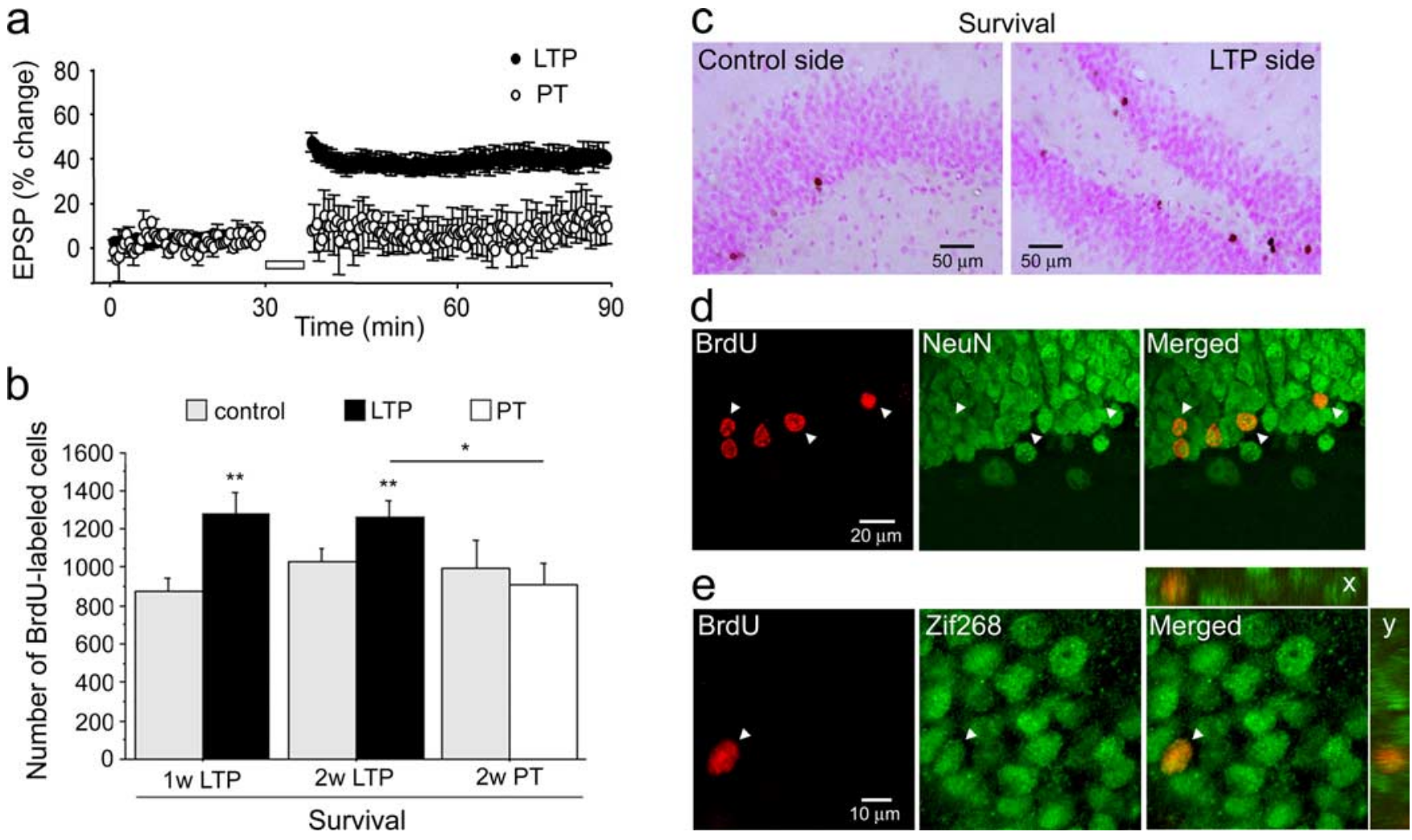

Figure 2. LTPenhances survival of recently born DGCs. $\boldsymbol{a}$, Time course of LTP of the fEPSP slope, as in Figure 1a. $\boldsymbol{b}$, Average total number of BrdU-labeled cells (mean \pm SEM) in the DG from control, and LTP or PT sides. LTP was induced 1 or 2 weeks after BrdU injections and rats were killed at the survival time of 4 weeks post-BrdU. ${ }^{*} p<0.05 ; *{ }^{* *} p<0.01$. c, Representative BrdU-stained sections (control and LTP sides, see Fig. 1b) at 4 week survival time from an animal in which LTP was induced 2 weeks after BrdU injections. $d$, Confocal images of double-stained cells for BrdU (red) and NeuN (green) 4 weeks after BrdU injections illustrating colocalization (merged) in DGCs (arrowheads). $\boldsymbol{e}$, Confocal images of double-immunostaining for BrdU (red) and Zif268 (green) $2 \mathrm{~h}$ after LTP induction from an animal injected with BrdU 2 weeks before LTP induction.

Two-week-old but not 1-week-old newborn neurons express Zif268 after induction of LTP

One key molecular mechanism underlying the expression of LTP in neurons is the transactivation of immediate early genes encoding inducible transcription factors, leading to downstream gene transcription and synthesis of proteins that modify synaptic transmission. One such transcription factor closely associated with hippocampal LTP is Zif268 (Jones et al., 2001). In DGCs, Zif268 is not constitutively expressed but is robustly induced by LTP, with protein levels peaking $\sim 2 \mathrm{~h}$ post-tetanus (Davis et al., 2003). Thus, to examine whether newborn DGCs aged 1-2 weeks were subject to potentiation by perforant path tetanization, sections from rats killed $2 \mathrm{~h}$ after LTP were analyzed for coexpression of BrdU and Zif268. In control sides from both groups (1 week, $n=3 ; 2$ weeks, $n=5$ ), there was no expression of Zif268 in DGCs, as expected, and in no case did we find BrdU colocalizing with Zif268, suggesting that 1- and 2-week-old cells do not express Zif268 constitutively. When LTP was induced, Zif268 was massively expressed in mature, non-BrdU-labeled cells throughout the dorsal DG (Fig. 2e) on the LTP side only. However, we found no evidence that 1-week-old BrdU-positive cell coexpressed Zif268 after LTP induction. In contrast, of 991 BrdUpositive cells examined, we found that $28 \pm 5 \%$ of 2 -week-old cells coexpressed Zif268 $2 \mathrm{~h}$ after LTP induction (Fig. 2e), suggesting that a fraction of these cells have undergone potentiation. BrdU/Zif268 coexpression was confined to the LTP side, indicating that Zif268 expression in 2-week-old cells is caused by LTP induction and not to developmentally regulated expression. These data show that after a 2 week maturation pe- riod, newborn DGCs participate in activity-dependent Zif268 expression after LTP.

\section{Discussion}

The results presented here demonstrate, first, that the induction of LTP at perforant path inputs to the DG in vivo promotes proliferation of progenitor cells in the following days without affecting neuronal differentiation; second, this proneurogenic effect of LTP results in a net increase in the proportion of cells surviving 4 weeks later; and third, the induction of LTP promotes survival of 1- to 2-week-old DGCs. Control experiments indicate these effects are likely to be caused by strong depolarization associated with electrically induced LTP and not to stimulus-driven synaptic activation of granule cells.

The induction of LTP at perforant path-DGC synapses requires activation of the NMDA receptor (NMDAR) (Bliss and Collingridge, 1993). In view of reports indicating that blocking NMDAR promotes adult neurogenesis (Cameron et al., 1995; Okuyama et al., 2004) it is surprising that LTP enhanced the rate of neurogenesis. However, our results are consistent with recent findings showing strong depolarization or glutamate activation can impinge on proliferating adult DG precursor cells and, in vivo, increase proliferating activity via L-type $\mathrm{Ca}^{2+}$ channels in an NMDAR-dependent manner (Deisseroth et al., 2004). Thus, the effect of blocking NMDAR may not mirror that of a more locally restricted NMDAR activation as brought about by LTP of one afferent system. After NMDAR activation, LTP triggers activation of many signaling cascades leading to channel and receptor modification, gene transcription, and protein synthesis. Al- 
though the present results do not allow any conclusion on the mechanisms mediating the neurogenic effect of LTP, they suggest that the induction of LTP can generate promitotic signals. Enhancement of mitotic activity was, however, detected at a relatively prolonged period after LTP induction $(4 \mathrm{~d})$. No report to date has shown that LTP can result in the production of neurogenic signals for several days. Thus, our results favor the view that any signal generated by LTP within the induction period or the following hours may initially promote cell divisions that would subsequently increase incrementally.

One possibility is mediation by secreted molecules from surrounding mature neurons undergoing LTP or glial cells. The molecular mechanisms regulating cell proliferation in the adult DG are, however, unclear. Newborn DGCs express pCREB, which has been implicated in maturation and survival (Nakagawa et al., 2002; Fujioka et al., 2004); however, we found no evidence for a specific activation of pCREB in newborn cells after LTP. Other putative mitotic signals could be involved, including nitric oxide (Zhang et al., 2001), Wnt (Lie et al., 2005), or Sonic hedgehog signaling (Lai et al., 2003). Whether these signaling mechanisms are activated in LTP will require additional studies.

Many newborn neurons die within a few weeks of their birth (Gould et al., 1999). Our results also show that LTP can promote later persistence ( 4 weeks) of proliferating cells. This indicates that increased neurogenesis is a persistent consequence of LTP induction, with the proviso that in this condition of previous induction of LTP, the increased population of surviving cells was presumably a direct consequence of LTP-induced enhancement in cell proliferation.

In contrast, when LTP was induced 1 or 2 weeks after BrdU injections, we found that LTP was largely beneficial to the survival, 4 weeks later, of these newly formed DGCs, demonstrating a direct enhancing effect of LTP on survival rate. Interestingly, this long-lasting effect parallels that recently found after learning (Leuner et al., 2004). Recent studies examining the maturation of newborn DGCs in adulthood suggest that 1-week-old neurons are immature, have few spines, and display depolarizing bicuculline-sensitive GABA currents before becoming sensitive to glutamatergic synaptic activation, which starts to be detected by only 2 weeks of age when they start forming spines and receive glutamatergic inputs (Esposito et al., 2005; Ge et al., 2006; Zhao et al., 2006). Dendritic and spine growth will continue thereafter while the neurons display mature excitability and become fully functional and synaptically integrated into corticohippocampal circuits. It is thus surprising that LTP can promote survival of newborn DGCs aged 1-2 weeks because these cells presumably do not receive, or receive only sparse (2 weeks) glutamatergic inputs. However, adult neuronal precursor cells may express NMDAR subunits (Nacher and McEwen, 2006), and SchmidtHieber et al. (2004) demonstrated that 1- to 3-week-old immature DGCs generate low-threshold $\mathrm{Ca}^{2+}$ spikes and have a lower threshold for LTP induced by bursts of perforant path stimuli paired with somatic current injection. The authors suggested that the specific membrane properties of newborn neurons may facilitate activation by sparse glutamatergic inputs. Consistent with this, our results show that a substantial fraction of 2-week-old newborn DGCs expressed Zif268 in response to LTP, confirming activity-dependent gene regulation in newborn DGCs (Jessberger and Kempermann, 2003). Thus, at least for 2-week-old neurons, direct potentiation of these immature neurons via perforant path inputs or by ambient glutamate remains an open possibility. However, we did not observe LTP-induced Zif268 expression in 1-week-old newborn cells despite increased sur- vival. Although we cannot exclude that the full repertoire of molecular mechanisms underlying late, transcription-dependent LTP may be nonfunctional in young neurons, this result supports the idea that LTP in surrounding mature cells provides a cellular/ molecular microenvironment that favors survival of recently born cells. For example, LTP is associated with release of many proteins including neurotrophic factors such as BDNF, and BDNF gene expression is enhanced for at least $24 \mathrm{~h}$ after LTP in the DG (Bramham and Messaoudi, 2005). Other, nonexclusive candidate mechanisms include extrasynaptic glutamate or GABA (Ge et al., 2006) from nearby neurons, which are released after LTP (Bliss and Collingridge, 1993). In any case, that 1- to 2-weekold neurons are sensitive to the occurrence of LTP in the DG long before their functional maturation is completed, is consistent with the idea derived from learning experiments that they already present a range of functional properties at early stages (Shors et al., 2001; Madsen et al., 2003).

Overall, the present findings suggest that the occurrence of LTP in the DG can promote both proliferation of newborn DGCs and survival of recently born DGCs. It is now widely recognized that adult neurogenesis can affect the expression of plasticity in this area (Schinder and Gage, 2004). The present findings that LTP promotes neurogenesis thus add a new prospect to the relationship between Hebbian synaptic plasticity and neurogenesis. They also present the intriguing hypothesis that the occurrence of activity-dependent synaptic plasticity in the DG during learning may provide signals for the promotion of learning-induced neurogenesis.

\section{References}

Bliss TVP, Collingridge GL (1993) A synaptic model of memory: long-term potentiation in the hippocampus. Nature 361:31-39.

Bramham CR, Messaoudi E (2005) BDNF function in adult synaptic plasticity: the synaptic consolidation hypothesis. Prog Neurobiol 76:99-125.

Bruel-Jungerman E, Laroche S, Rampon C (2005) New neurons in the dentate gyrus are involved in the expression of enhanced long-term memory following environmental enrichment. Eur J Neurosci 21:513-521.

Cameron HA, McEwen BS, Gould E (1995) Regulation of adult neurogenesis by excitatory input and NMDA receptor activation in the dentate gyrus. J Neurosci 15:4687-4692.

Davis S, Bozon B, Laroche S (2003) How necessary is the activation of the immediate early gene zif268 in synaptic plasticity and learning? Behav Brain Res 142:17-30.

Davis S, Vanhoutte P, Pages C, Caboche J, Laroche S (2000) The MAPK/ ERK cascade targets both Elk-1 and cAMP response element-binding protein to control long-term potentiation-dependent gene expression in the dentate gyrus in vivo. J Neurosci 20:4563-4572.

Deisseroth K, Singla S, Toda H, Monje M, Palmer TD, Malenka RC (2004) Excitation-neurogenesis coupling in adult neural stem/progenitor cells. Neuron 42:535-552.

Duffy SN, Craddock KJ, Abel T, Nguyen PV (2001) Environmental enrichment modifies the PKA-dependence of hippocampal LTP and improves hippocampus-dependent memory. Learn Mem 8:26-34.

Esposito MS, Piatti VC, Laplagne DA, Morgenstern NA, Ferrari CC, Pitossi FJ, Schinder AF (2005) Neuronal differentiation in the adult hippocampus recapitulates embryonic development. J Neurosci 25:10074-10086.

Fujioka T, Fujioka A, Duman RS (2004) Activation of cAMP signaling facilitates the morphological maturation of newborn neurons in adult hippocampus. J Neurosci 24:319-328.

Ge S, Goh EL, Sailor KA, Kitabatake Y, Ming GL, Song H (2006) GABA regulates synaptic integration of newly generated neurons in the adult brain. Nature 439:589-593.

Gould E, Beylin A, Tanapat P, Reeves A, Shors TJ (1999) Learning enhances adult neurogenesis in the hippocampal formation. Nat Neurosci 2:260-265.

Jessberger S, Kempermann G (2003) Adult-born hippocampal neurons mature into activity-dependent responsiveness. Eur J Neurosci 18:2707-2712. 
Jones MW, Errington ML, French PJ, Fine A, Bliss TVP, Garel S, Charnay P, Bozon B, Laroche S, Davis S (2001) A requirement for the immediate early gene Zif268 in the expression of late LTP and the consolidation of long-term memories. Nat Neurosci 4:289-296.

Kempermann G, Kuhn HG, Gage FH (1997) More hippocampal neurons in adult mice living in an enriched environment. Nature 386:493-495.

Kempermann G, Gast D, Kronenberg G, Yamaguchi M, Gage FH (2003) Early determination and long-term persistence of adult-generated new neurons in the hippocampus of mice. Development 130:391-399.

Kronenberg G, Reuter K, Steiner B, Brandt MD, Jessberger S, Yamaguchi M, Kempermann G (2003) Subpopulations of proliferating cells of the adult hippocampus respond differently to physiologic neurogenic stimuli. J Comp Neurol 467:455-463.

Lai K, Kaspar BK, Gage FH, Schaffer DV (2003) Sonic hedgehog regulates adult neural progenitor proliferation in vitro and in vivo. Nat Neurosci 6:21-27.

Leuner B, Mendolia-Loffredo S, Kozorovitskiy Y, Samburg D, Gould E, Shors TJ (2004) Learning enhances the survival of new neurons beyond the time when the hippocampus is required for memory. J Neurosci 24:7477-7481.

Lie DC, Colamarino SA, Song HJ, Desire L, Mira H, Consiglio A, Lein ES, Jessberger S, Lansford H, Dearie AR, Gage FH (2005) Wnt signalling regulates adult hippocampal neurogenesis. Nature 437:1370-1375.

Lynch MA (2004) Long-term potentiation and memory. Physiol Rev 84:87-136.

Madsen TM, Kristjansen PE, Bolwig TG, Wortwein G (2003) Arrested neuronal proliferation and impaired hippocampal function following fractionated brain irradiation in the adult rat. Neuroscience 119:635-642.

Martin SJ, Morris RG (2002) New life in an old idea: the synaptic plasticity and memory hypothesis revisited. Hippocampus 12:609-636.

Nacher J, McEwen BS (2006) The role of N-methyl-D-asparate receptors in neurogenesis. Hippocampus 16:267-270.

Nakagawa S, Kim JE, Lee R, Malberg JE, Chen J, Steffen C, Zhang YJ, Nestler
EJ, Duman RS (2002) Regulation of neurogenesis in adult mouse hippocampus by cAMP and the cAMP response element-binding protein. J Neurosci 22:3673-3682.

Okuyama N, Takagi N, Kawai T, Miyake-Takagi K, Takeo S (2004) Phosphorylation of extracellular-regulating kinase in NMDA receptor antagonist-induced newly generated neurons in the adult rat dentate gyrus. J Neurochem 88:717-725.

Schinder AF, Gage FH (2004) A hypothesis about the role of adult neurogenesis in hippocampal function. Physiology 19:253-261.

Schmidt-Hieber C, Jonas P, Bischofberger J (2004) Enhanced synaptic plasticity in newly generated granule cells of the adult hippocampus. Nature 429:184-187.

Shors TJ, Miesegaes G, Beylin A, Zhao M, Rydel T, Gould E (2001) Neurogenesis in the adult is involved in the formation of trace memories. Nature 410:372-376.

Snyder JS, Hong NS, McDonald RJ, Wojtowicz JM (2005) A role for adult neurogenesis in spatial long-term memory. Neuroscience 130:843-852.

van Praag H, Christie BR, Sejnowski TJ, Gage FH (1999) Running enhances neurogenesis, learning, and long-term potentiation in mice. Proc Natl Acad Sci USA 96:13427-13431.

van Praag H, Schinder AF, Christie BR, Toni N, Palmer TD, Gage FH (2002) Functional neurogenesis in the adult hippocampus. Nature 415:1030-1034.

Winocur G, Wojtowicz JM, Sekeres M, Snyder JS, Wang S (2006) Inhibition of neurogenesis interferes with hippocampus-dependent memory function. Hippocampus 16:296-304.

Zhang R, Zhang L, Zhang Z, Wang Y, Lu M, Lapointe M, Chopp M (2001) A nitric oxide donor induces neurogenesis and reduces functional deficits after stroke in rats. Ann Neurol 50:602-611.

Zhao C, Teng EM, Summers Jr RG, Ming GL, Gage FH (2006) Distinct morphological stages of dentate granule neuron maturation in the adult mouse hippocampus. J Neurosci 26:3-11. 